\title{
The PAIR-R24M Dataset for Multi-animal 3D Pose Estimation
}

\author{
Jesse D. Marshall* \\ Organismic and Evolutionary Biology \\ Harvard University \\ Cambridge, MA 02138 \\ jesse_d_marshall@fas.harvard.edu
}

Amanda Gellis

Organismic and Evolutionary Biology

Harvard University

Cambridge, MA 02138

agellis@fas. harvard. edu

\author{
Bence P. Ölveczky \\ Organismic and Evolutionary Biology \\ Harvard University \\ Cambridge, MA 02138 \\ olveczky@fas . harvard . edu
}

\author{
Ugne Klibaite* \\ Organismic and Evolutionary Biology \\ Harvard University \\ Cambridge, MA 02138 \\ klibaite@fas.harvard.edu
}

\author{
Diego E. Aldarondo \\ Organismic and Evolutionary Biology \\ Harvard University \\ Cambridge, MA 02138 \\ diegoaldarondo@g . harvard.edu \\ Timothy W. Dunn \\ Department of Biomedical Engineering \\ Duke University \\ Durham, NC 27708 \\ timothy.dunn@duke.edu
}

\begin{abstract}
Understanding the biological basis of social and collective behaviors in animals is a key goal of the life sciences, and may yield important insights for engineering intelligent multi-agent systems. A critical step in interrogating the mechanisms underlying social behaviors is a precise readout of the 3D pose of interacting animals. While approaches for multi-animal pose estimation are beginning to emerge, they remain challenging to compare due to the lack of standardized training and benchmark datasets. Here we introduce the PAIR-R24M (Paired Acquisition of Interacting oRganisms - Rat) dataset for multi-animal 3D pose estimation, which contains 24.3 million frames of RGB video and 3D ground-truth motion capture of dyadic interactions in laboratory rats. PAIR-R24M contains data from 18 distinct pairs of rats and 24 different viewpoints. We annotated the data with 11 behavioral labels and 3 interaction categories to facilitate benchmarking in rare but challenging behaviors. To establish a baseline for markerless multi-animal 3D pose estimation, we developed a multi-animal extension of DANNCE, a recently published network for $3 \mathrm{D}$ pose estimation in freely behaving laboratory animals. As the first large multi-animal 3D pose estimation dataset, PAIR-R24M will help advance 3D animal tracking approaches and aid in elucidating the neural basis of social behaviors.
\end{abstract}

\footnotetext{
${ }^{*}$ Equal contribution.
} 


\section{Introduction}

Social behaviors are core components of an animal's behavioral repertoire. Understanding their neural, biological, and evolutionary basis has long been a focus of the life sciences [1,2] and may inform treatments for psychiatric diseases, such as autism spectrum disorder and schizophrenia, where social interactions are impaired [3,4].

Precisely phenotyping social behaviors and identifying their neural basis requires reliable and quantitative measures of social behavior in animal models [5]. Currently, studies largely rely on scoring performance in highly structured assays, for instance the tube test, 3 chamber test, or resident-intruder test [6]. While these provide interpretable readouts, they are ethologically limited and compress complex behavioral processes into scalar variables of questionable biological significance [7]. In contrast, assays in unrestrained animals that use computer vision and behavioral classification offer the ability to profile a richer range of social behaviors between animals, but are more challenging to quantify and interpret [8-12].

To improve behavioral quantification, convolutional neural networks for automated detection of an animal's 2D pose [13-15], and more recently 3D pose [16-18], have been developed. However, in comparison to single animal tracking, methods for multi-animal postural tracking, especially in $3 \mathrm{D}$, are only beginning to emerge. Existing $2 \mathrm{D}$ pose recognition techniques employ a mixture of 'top-down' multi-animal tracking, in which pose is reconstructed within identified bounding boxes of multiple animals (e.g [8,14]) and 'bottom up' architectures that first detect all body landmarks and then assign them to animals [19-21]. Both top-down and bottom-up multi-animal tracking approaches are promising, but need substantial amounts of training data to accurately track animal pose in the face of challenging occlusions generated by socially interacting animals.

Development of new data-efficient and occlusion-robust multi-animal tracking approaches requires standardized pose estimation datasets and benchmarks, which do not exist in 3D. To address this, we introduce PAIR-R24M, a novel dataset relating multi-view color video and ground-truth 3D kinematics in behaving rats. We collected over 24 million frames of $30 \mathrm{~Hz}$ color video across 24 camera views in 18 different pairs of rats interacting in a behavioral arena. In each frame, a motion capture system provides the 3D positions of 12 body landmarks on each individually identified animal, describing the movement of its head, trunk, shoulders, and hips. Each frame is associated with a behavioral label, denoting which of 11 behavioral categories and 3 inter-animal interaction categories it matches best. These labels can be used to balance datasets during training, rigorously assess pose estimation performance over a wide variety of poses, provide labels for action recognition approaches, and perform detailed analyses of behavioral patterns.

\section{Related Work}

\subsection{Datasets for single and multi-animal 3D pose}

There exists a small collection of publicly available 3D animal pose benchmark datasets. The Acino dataset contains 7,588 frames of hand-labeled 3D poses (20 keypoints) from cheetahs, capturing mostly running behaviors [22]. The Open Monkey Studio dataset contains 195,228 hand-labeled frames (13 keypoints) of macaques in a large, enriched enclosure across 62 camera views [16]. Two other approaches use motion capture systems to provide expanded 3D ground-truth datasets. RGBDDog includes 3D keypoint data (63-82 keypoints from motion capture) and depth maps along with 8-10 RGB video views in canines, although is limited to 5 behaviors [23]. Rat 7M contains nearly 7 million frames and 3D keypoints across a wide range of rat poses, providing a powerful substrate for training and testing algorithms in rodents, the most common model organisms in biomedicine [18]. While valuable, each of the datasets is limited to individual animals.

Thus far, multi-animal datasets exist only for 2D. Graving et al. released videos and 2D annotations for large groups of locusts and zebras filmed from a single top-down view [14], providing valuable datasets for benchmarking 2D collective behavior tracking algorithms. Pereira et al. published a set of labeled fruit fly courtship data [20]. Lauer et al. released annotated multi-animal datasets from mice, mouse pups, marmoset, and zebrafish [21]. By far the most extensive multi-animal 2D dataset is CalMS21, which was released as part of the Multi-Agent Behavior Challenge 2021 and consists of 6 million frames of unlabeled and over 1 million frames of tracked poses and behavioral annotations of pairs of interacting mice [24]. 
In the more mature field of 3D human pose estimation, many multi-human 3D datasets are available, which vary broadly in the number of behaviors tracked, number of cameras used, means of marker tracking, and environmental context. The CMU Panoptic dataset provides 480 camera views during a wide range of social behaviors in a laboratory environment, with $3 \mathrm{D}$ poses obtained via pose estimation [25]. The Campus, Shelf (manually annotated) and MuPoTS-3D (derived from pose estimation) datasets offer 3D poses and multi-view video in real-world scenes [26,27], while 3DPW offers monocular footage with 3D pose labels derived from inertial measurement units [28]. The MuCo-3DHP dataset [27] is a large, multi-human 3D dataset generated by splicing together individual subjects, and their ground-truth markerless annotations, from the expansive MPI-INF-3DHP dataset [29]. Other benchmark datasets exist in specific domains, such as stores [30] and operating rooms [31]. Others use synthetically rendered humans [32-36] or body surfaces [37]. Together these datasets have fueled a productive era of 3D pose tracking, but their domain is drastically different from laboratory animals. Developing the type of 3D animal tracking algorithms required to accelerate progress in neuroscience, biomedicine, and ecology will require in-domain datasets that permit relevant training and benchmarking over a diversity of body plans and behaviors.

\subsection{Algorithms and benchmarks for animal 3D Pose Estimation}

To our knowledge there is only one example of multi-animal 3D pose estimation in the literature [16], likely due to the lack of large training and benchmark datasets in this domain. There are several existing algorithms for 3D pose in individual animals. DANNCE [18] and Freipose [17] use volumetric representations of multi-view inputs to combine image features across cameras and enable 3D supervision, similar to the current state-of-the-art for multi-view human pose [38]. 3D DeepLabCut $[22,39]$ uses triangulation of 2D detections across multiple views, which GIMBAL [40] and Anipose [41] further refine using spatiotemporal constraints. Open Monkey Studio uses a triangulation-based method but with a larger set of cameras, and in addition to using spatiotemporal constraints, makes use of reprojections into unlabeled views to increased their labeled training pool [16]. DeepFly3D uses triangulation, bundle adjustment, and pictorial structures to provide robust 3D pose estimation in tethered flies [42]. For monocular 3D pose estimation, "lifting" approaches using a fully connected network to infer 3D pose from 2D estimates $[43,44]$ have been extended from work in humans [45] to tethered flies and lab mammals. In addition to lifting, Bolaños et al. [43] use synthetic data to improve 3D pose detection in restrained mice. As of yet, none of these methods have been extended to multi-animal 3D pose estimation. In this study we extend the DANNCE volumetric approach because it has demonstrated superior performance on rodents compared to multi-view triangulation, and also because multi-view triangulation would be further complicated by errors in multi-animal identity tracking.

\subsection{Multi-animal action recognition}

We follow the lead of human 3D pose datasets and group our data into standardized behavioral categories to aid the training and benchmarking of pose-estimation and action-recognition algorithms. However, unlike traditional 3D pose datasets acquired using human actors given explicit instructions, here we needed to infer behavioral categories from movement by extending 3D action recognition methods to the multi-animal setting. Multi-animal action recognition has remained challenging due to a lack of ground-truth and, relatedly, a lack of intuition about the definitions and structure of animal behavior, especially in social contexts. Existing methods for multi-animal action recognition employ supervised learning using human-labeled behavior categories such as mounting or attacking, classified using a variety of features describing behavior: pixels [46], the set of 2D body landmarks visible from a single top-down views [8,24], hand-designed features of 2D body landmarks [47] (sometimes supplemented with depth imaging information [48]), shapes fit to 2D or 3D body contours [9, 49], or quantities derived from movement trajectories, such as velocity and heading direction [50]. Other methods use unsupervised learning techniques, again on a range of behavioral features: pixels [51], $2 \mathrm{D}$ pose features [12] or both [30]. While no approach has performed unsupervised analysis of multiple animals using 3D pose, Marshall et al. [52] designed an approach for identifying behaviors in single animals based on 3D pose features. Here we extend this approach to multiple animals, and create inter-individual features to define new interaction behavioral categories. This straightforward, yet effective, unsupervised action recognition approach allows us to segment and balance the PAIRR24M dataset and introduce a foundational algorithm applicable to new multi-subject 3D pose data across species. 


\section{Dataset and Benchmark}

\subsection{The PAIR-R24M dataset}

To collect the PAIR-R24M dataset we used CAPTURE, a technique that uses body piercing to chronically attach retro-reflective markers to small animals, allowing their pose to be reconstructed using motion capture [52]. We attached 12 markers to the dorsal surface of each animal at identical locations to label their head, trunk, hips, and shoulders. We additionally added 1-2 markers to the head and trunk of animals to differentiate individuals. If interacting animals bore identical marker sets, we masked a marker on the head using whiteout to disambiguate them.

We used a 12 camera motion capture array to record the position of the markers at $300 \mathrm{~Hz}$ with sub-mm precision (Fig. 1A). We used commercial Cortex (Motion Analysis) software, which utilizes pairwise distances between markers and a parametric body model, to assign marker identities to each animal. We concurrently recorded animals at $30 \mathrm{~Hz}$ using 6 RGB video cameras. We calibrated the video cameras into the same world coordinate system as the motion capture array to automatically label video frames by projecting the 3D marker positions.

We then performed simultaneous CAPTURE and video recordings for 18 pairs of animals $(n=7$ subjects bearing markers, $n=2$ markerless subjects), for 1 hour each (108,000 timepoints). To increase viewpoint diversity, we moved each of the video cameras to 4 different locations across recordings (Fig. 1B). On a subset of camera views and frames in which animals were rapidly moving, we noted discrepancies between motion capture and video due to slight errors in synchronization and calibration (Appendix 3). While these errors could in the long term pose limits in the precision of the dataset as a benchmark, they occur on a limited subset of frames, and similar discrepancies exist in commonly used human 3D pose datasets [38].

We recorded from a subset of animal pairs in each recording condition, yielding a total of 26 hours of data of paired animals bearing markers. We also recorded 14 hours of data from animals bearing markers when paired with animals not bearing markers, to add additional markerless video frames to the dataset. These single-markerset recordings also allowed us to assess the fidelity of animal identity assignment in the dataset. Head segment lengths, which were constant within subjects but differed slightly between subjects due to small changes in head marker placement during headcap construction, were stable across individual animals when compared over single- and double-markerset paired recordings (Appendix 4). Additionally, we recorded from each subject alone for 30 minutes to facilitate the construction of single animal tracking models, and recorded from individual and paired animals not bearing markers. Single animal and paired markerless video recordings are not included in the present dataset but may be added at a later data to facilitate transfer and benchmarking of semi-supervised tracking approaches.

Occasionally, self-, animal-animal, or environmental occlusions prevented 3D marker tracking by the motion capture system. As most of these periods were temporally succinct, we imputed missing data using linear interpolation within an egocentrically aligned reference frame anchored on the animal's center of mass and rotated to place the front of the animal's spine along the y-axis. The center of mass and orientation of the animals were estimated from the remaining markers if spine markers were absent. We also sometimes observed other errors where the motion capture system incorrectly assigned marker position. We addressed incorrect assignment by flagging potential errors using a $4 \sigma$ threshold on z-scores of inter-marker distance, although we note these frames still appeared to possess accurate behavioral categorization. Our official 24M dataset size is calculated after excluding any frame with at least one flagged marker. In the released dataset, we provide all recorded frames, together with z-scores for each marker, permitting researchers to use partially tracked frames if desired.

\subsection{Action recognition}

The performance of human and animal pose tracking algorithms can vary widely depending on the behaviors animals perform - for instance highly-occlusive rodent grooming behaviors are often challenging to reconstruct - making it important to assess the performance of tracking algorithms in an action-specific manner. There remains no standard taxonomy of rodent behaviors [53], and there is often disagreement among human observers about what defines a behavior and when they begin and end (e.g [24,54]). We therefore used an unsupervised approach to identify behaviors by 
A

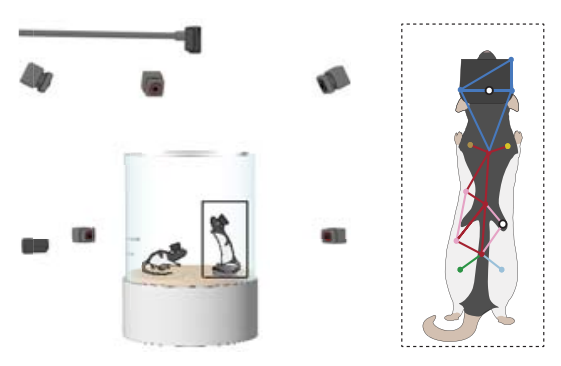

C

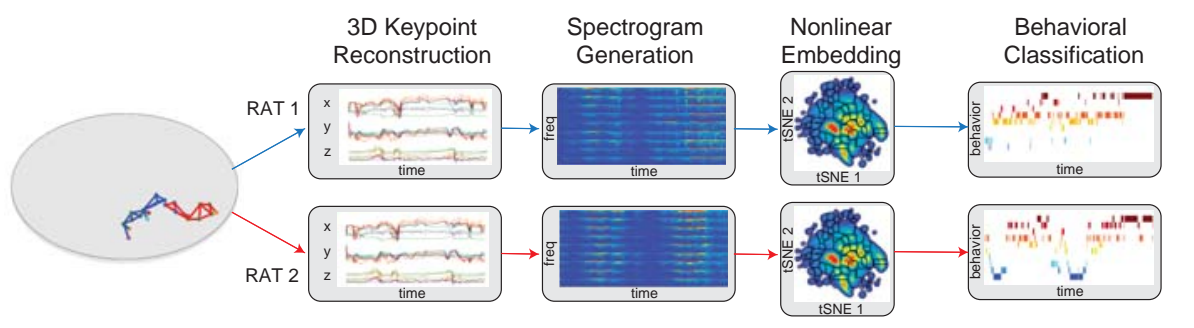

D

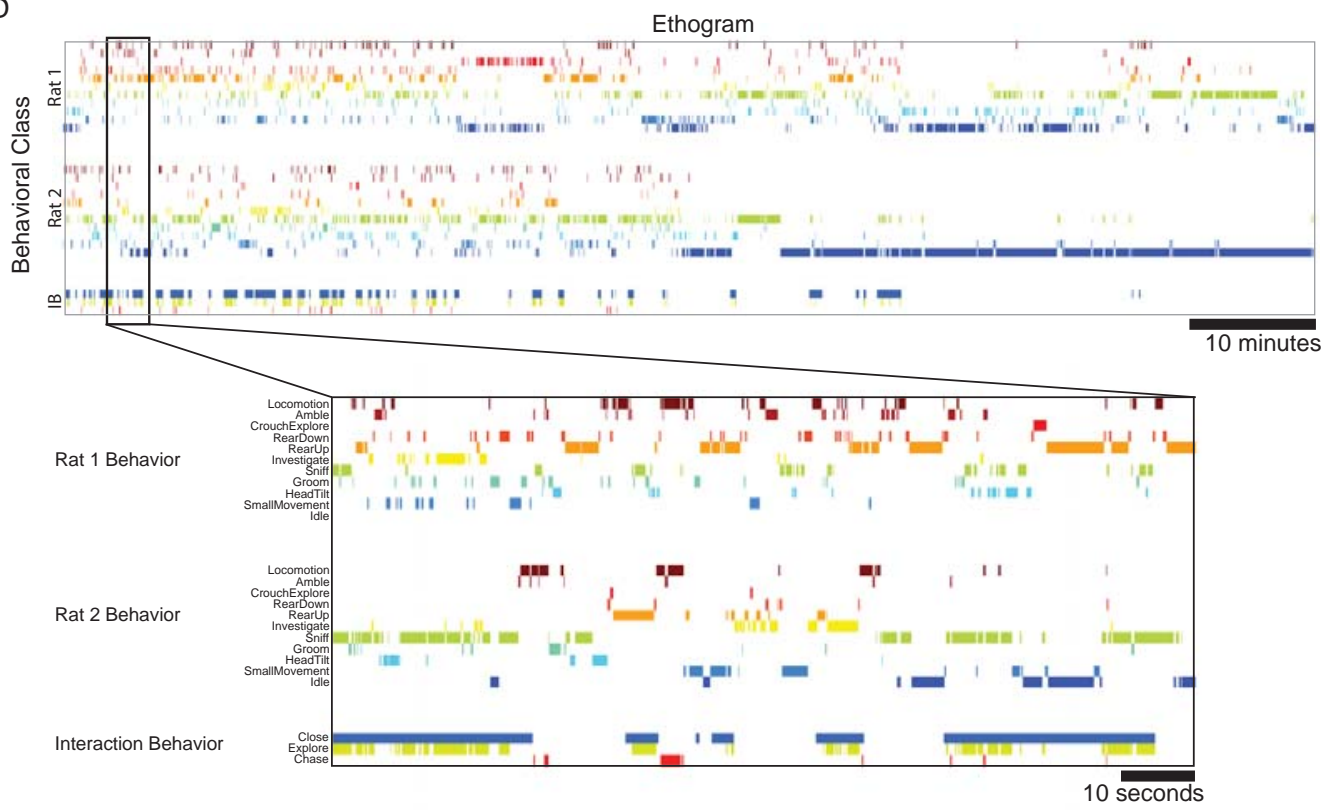

B



Figure 1: (A) Schematic of the recording arena with two interacting rats surrounded by motion capture and video cameras. Inset: location of the recording markers used on the animal's body. White markers indicate markers that were tracked in a subset of animals to distinguish between animal pairs, but are not used for further analysis. (B) Location of the video camera positions relative to the recording arena across all experiments. Each camera was moved to 4 different locations during acquisition of the dataset, but there were additional minor shifts in position across recordings (42 total positions across all cameras). (C) Analysis pipeline schematic for applying behavioral labels given 12-point skeletons obtained from motion capture recordings. (D) Ethograms for individual and interaction behavioral categories for a sample 1-hour movie. Expanded region corresponds to a two-minute behavioral bout. Example Movies. 


\begin{tabular}{lllllllll}
\hline & Subj. 1 & Subj. 2 & Subj. 3 & Subj. 4 & Subj. 5 & Subj. 9 & Subj. 10 & All \\
\hline Pairs & 6 & 6 & 6 & 6 & 6 & 2 & 2 & 18 \\
CL $_{50}$ & 156 & 110 & 90 & 134 & 240 & 178 & 128 & 138 \\
CL $_{95}$ & 2642 & 1200 & 1300 & 1281 & 2433 & 1676 & 1283 & 2593 \\
IB1 (Close) & $1.42 \mathrm{M}$ & $1.19 \mathrm{M}$ & $1.32 \mathrm{M}$ & $1.23 \mathrm{M}$ & $872 \mathrm{k}$ & $307 \mathrm{k}$ & $307 \mathrm{k}$ & $3.32 \mathrm{M}$ \\
IB2 (Explore) & $797 \mathrm{k}$ & $763 \mathrm{k}$ & $823 \mathrm{k}$ & $752 \mathrm{k}$ & $448 \mathrm{k}$ & $164 \mathrm{k}$ & $164 \mathrm{k}$ & $1.96 \mathrm{M}$ \\
IB3 (Chase) & $29.6 \mathrm{k}$ & $32.4 \mathrm{k}$ & $33.4 \mathrm{k}$ & $42.1 \mathrm{k}$ & $24.2 \mathrm{k}$ & $21.8 \mathrm{k}$ & $21.8 \mathrm{k}$ & $103 \mathrm{k}$ \\
B1 (Idle) & $2.69 \mathrm{M}$ & $1.95 \mathrm{M}$ & $1.84 \mathrm{M}$ & $1.80 \mathrm{M}$ & $2.52 \mathrm{M}$ & $377 \mathrm{k}$ & $1.01 \mathrm{M}$ & $12.2 \mathrm{M}$ \\
B2 (SmallMovement) & $897 \mathrm{k}$ & $707 \mathrm{k}$ & $484 \mathrm{k}$ & $488 \mathrm{k}$ & $394 \mathrm{k}$ & $224 \mathrm{k}$ & $105 \mathrm{k}$ & $3.30 \mathrm{M}$ \\
B3 (HeadTilt) & $399 \mathrm{k}$ & $260 \mathrm{k}$ & $268 \mathrm{k}$ & $223 \mathrm{k}$ & $428 \mathrm{k}$ & $166 \mathrm{k}$ & $183 \mathrm{k}$ & $1.93 \mathrm{M}$ \\
B4 (Groom) & $458 \mathrm{k}$ & $606 \mathrm{k}$ & $319 \mathrm{k}$ & $302 \mathrm{k}$ & $290 \mathrm{k}$ & $210 \mathrm{k}$ & $120 \mathrm{k}$ & $2.31 \mathrm{M}$ \\
B5 (Sniff) & $1.32 \mathrm{M}$ & $1.71 \mathrm{M}$ & $1.01 \mathrm{M}$ & $1.25 \mathrm{M}$ & $1.50 \mathrm{M}$ & $421 \mathrm{k}$ & $372 \mathrm{k}$ & $7.59 \mathrm{M}$ \\
B6 (Investigate) & $535 \mathrm{k}$ & $438 \mathrm{k}$ & $246 \mathrm{k}$ & $319 \mathrm{k}$ & $155 \mathrm{k}$ & $169 \mathrm{k}$ & $60.4 \mathrm{k}$ & $1.92 \mathrm{M}$ \\
B7 (RearUp) & $896 \mathrm{k}$ & $736 \mathrm{k}$ & $787 \mathrm{k}$ & $638 \mathrm{k}$ & $253 \mathrm{k}$ & $236 \mathrm{k}$ & $119 \mathrm{k}$ & $3.66 \mathrm{M}$ \\
B8 (RearDown) & $223 \mathrm{k}$ & $215 \mathrm{k}$ & $200 \mathrm{k}$ & $228 \mathrm{k}$ & $126 \mathrm{k}$ & $153 \mathrm{k}$ & $90.6 \mathrm{k}$ & $1.24 \mathrm{M}$ \\
B9 (CrouchExplore) & $230 \mathrm{k}$ & $67.5 \mathrm{k}$ & $206 \mathrm{k}$ & $122 \mathrm{k}$ & $34.6 \mathrm{k}$ & $65.5 \mathrm{k}$ & $28.4 \mathrm{k}$ & $755 \mathrm{k}$ \\
B10 (Amble) & $101 \mathrm{k}$ & $101 \mathrm{k}$ & $90.5 \mathrm{k}$ & $109 \mathrm{k}$ & $111 \mathrm{k}$ & $61.8 \mathrm{k}$ & $54.4 \mathrm{k}$ & $628 \mathrm{k}$ \\
B11 (Locomotion) & $235 \mathrm{k}$ & $214 \mathrm{k}$ & $202 \mathrm{k}$ & $222 \mathrm{k}$ & $180 \mathrm{k}$ & $288 \mathrm{k}$ & $138 \mathrm{k}$ & $1.48 \mathrm{M}$ \\
Total Frames & $7.98 \mathrm{M}$ & $7.00 \mathrm{M}$ & $5.65 \mathrm{M}$ & $5.71 \mathrm{M}$ & $5.99 \mathrm{M}$ & $2.37 \mathrm{M}$ & $2.28 \mathrm{M}$ & $24.3 \mathrm{M}$ \\
\hline
\end{tabular}

Table 1: Recording summary statistics for all animal subjects. Pairs is the total number of unique animal pairs recorded for each subject. $\left(\mathrm{CL}_{50}\right) 50$ th percentile of contiguous clip length (in frames) after excluding frames with at least one poorly tracked marker in both animals; (CL95) 95th percentile of contiguous clip length (in frames); (IBx) frames for interaction behaviors; (Bx) frames for individual behaviors. The "All" column tallies over unique items (e.g. Subj. $2+$ Subj. 1 IB only counted once).

first clustering pose dynamics in a reduced dimensional behavioral feature space, and then manually inspecting samples from each cluster to assign cluster names post hoc, following previously published approaches $[52,55]$. To cluster the animals' behavior, we first performed principal component analysis on the all-to-all marker distances across all frames. We applied a Morlet wavelet transform to the top 10 principal components at 25 dyadically spaced frequencies from $0.5-20 \mathrm{~Hz}$. These features, along with the z-heights and local smoothed velocities of each marker, composed a feature vector. To balance the clustering, we applied tSNE separately to each recording and sampled 1,000 frames distributed evenly across the behavioral embedding of each reduced dataset [12]. We then concatenated the sampled frames from each dataset and embedded them with tSNE, resulting in a comprehensive, balanced embedding space of all animal behavior in the dataset. We then re-embedded wavelet values from each movie using convex optimization, as described in [55], transformed the map into a density distribution after smoothing it with a Gaussian kernel, and applied a watershed transform to divide the data into discrete clusters.

The number of behavioral clusters identified in the embedding space can be varied by changing the density kernel used to create the space. We provide two resolutions of behavioral labeling in the dataset. First, a set of 11 coarse behavioral categories that can be used to balance the dataset and benchmark algorithms across different behaviors. Second, a set of 84 fine behavioral categories that can be used for a more detailed analysis of the animal's behavior.

The coarse behavioral categories reflected common classes of rodent behavior, including rearing, locomotion, and investigation (Fig. 2), each of which results from a manual clustering of fine-grained clusters. Within these fine behavioral categories across the full dataset, behaviors varied in frequency by several orders of magnitude, from 6,000 to 6,000,000 time-points (Fine Behavior 62 - a sideto-side head sweep vs. Fine Behavior 35 - a high-frequency sniff). This class imbalance highlights the importance of obtaining large datasets to train and benchmark behavioral tracking algorithms, especially if algorithm performance on rare behaviors is desired.

To further isolate different classes of inter-animal interactions, we further divided periods in which animals' centroids were within one body length of one another $(200 \mathrm{~mm})$ into three different interaction behavioral categories: synchronized locomotion ("Chase"), stationary exploration ("Explore"; when both animals were in any coarse behavioral category among HeadTilt, Groom, Sniff, Investigate, Rears, and CrouchExplore), or other times when animals were adjacent ("Close"). Because 
bioRxiv preprint doi: https://doi.org/10.1101/2021.11.23.469743; this version posted November 25, 2021. The copyright holder for this preprint (which was not certified by peer review) is the author/funder, who has granted bioRxiv a license to display the preprint in perpetuity. It is made available under aCC-BY 4.0 International license.

inter-animal interactions contain numerous occlusions, they represent a challenging use case for multi-animal tracking algorithms. The over 5.3 million frames of animal interactions we provide here provide an ample diversity of frames to train and benchmark new pose tracking algorithms in social settings.
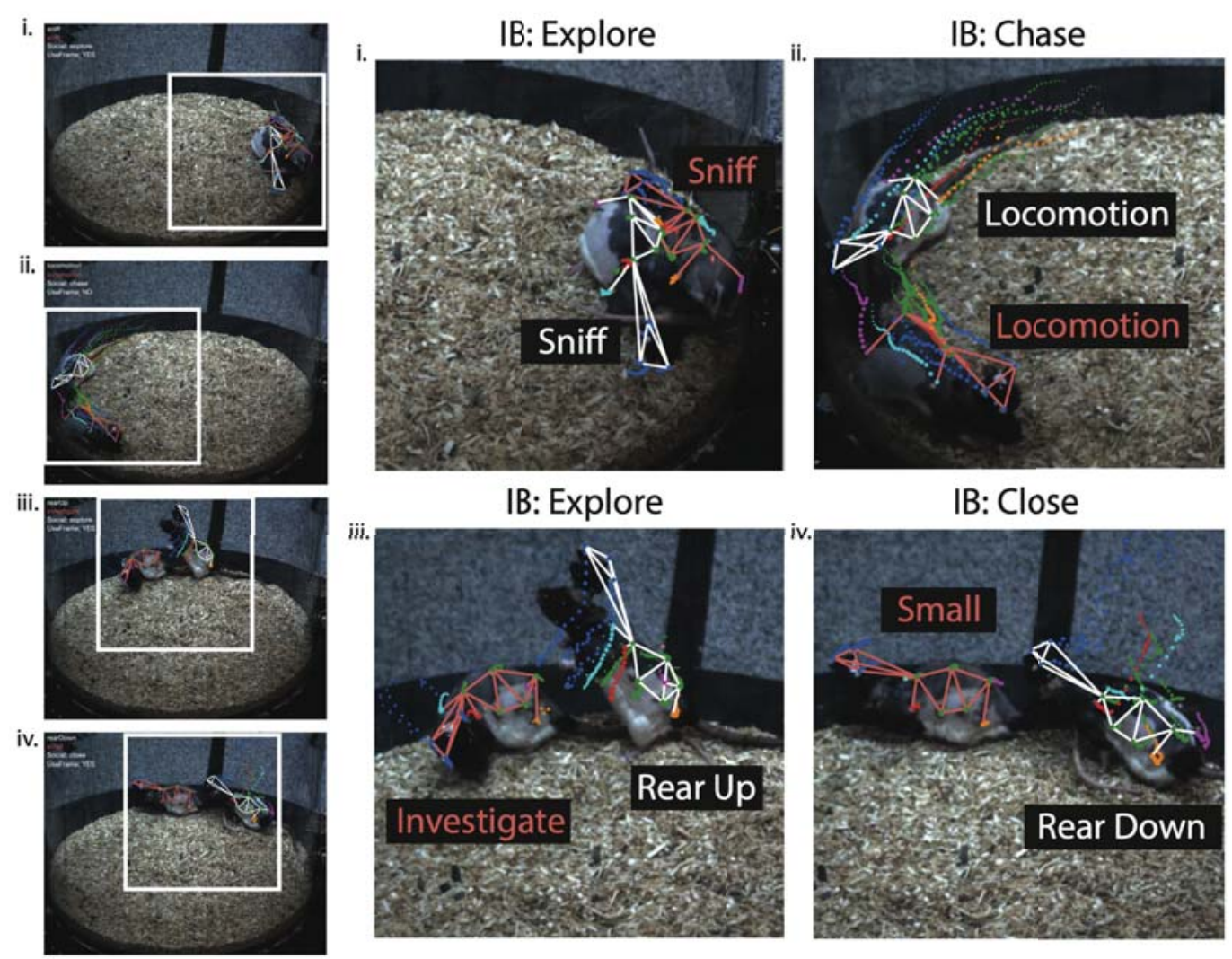

Figure 2: Example reprojections of ground-truth motion capture onto single camera views, shown for specific behavioral categories (pink and white labels corresponding to each rat skeleton) and interaction behavior categories (IB). Trailing points illustrate past 1-second trajectories for each marker. Example Movies.

The video frames, 3D pose estimates, and behavioral annotations are continuous in time, with only moderate interruptions in pose estimates due to flagged tracking errors. The median length of continuously tracked snippets is 138 frames ( $4.5 \mathrm{~s}$ ), with a long tail such that $5 \%$ of all continuous snippets are greater than $86 \mathrm{~s}$ in length (Table 1). This will be useful both for benchmarking videobased pose tracking algorithms that use local temporal information [56], as well as building statistical models of single and multi-animal behavior [57,58]. As an example of their use for analyzing the mathematical structure of behavior, we can visualize the ethograms of each animal's behavior, which show that animals transition over many individual and interacting behaviors during a recording session (Fig. 1D).

\subsection{DANNCE benchmark}

To establish baseline benchmarks for pose estimation to which future algorithms should be compared, we used a multi-animal extension of DANNCE [18], the current state-of-the-art for rat 3D pose estimation. Because DANNCE's standard mechanism is to encapsulate a subject in a 3D volumetric bounding box via geometric sampling of multi-view image content, multi-animal inference was performed simply by running each animal's 3D volume through the network independently (see Appendix 5 for details). When animals are separated in space, such that their 3D volumes are 
non-overlapping, this approach trivially reduces to the single animal case. When animals are nearby and overlapping, however, DANNCE must overcome significant animal-animal occlusion and infer correct landmark-subject associations. Our dataset provides a large library of interacting behavior examples that DANNCE, and other approaches, can use to learn social-specific poses and complex, multi-animal image features.

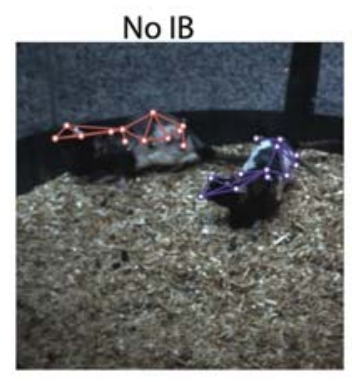

IB: Explore

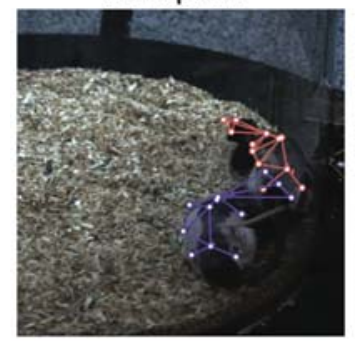

No IB

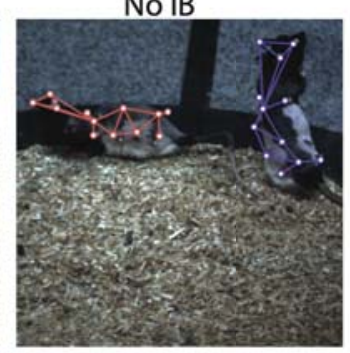

IB: Explore

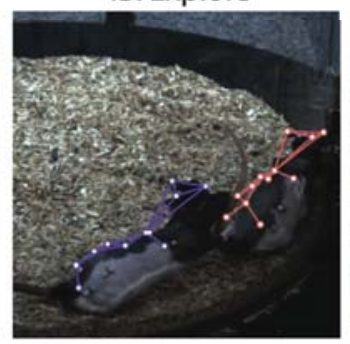

IB: Chase

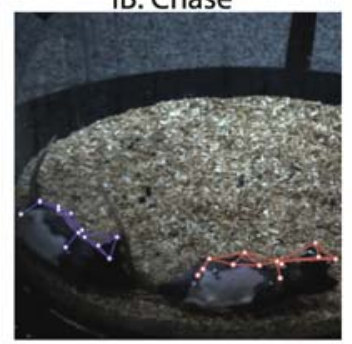

IB: Explore



IB: Close

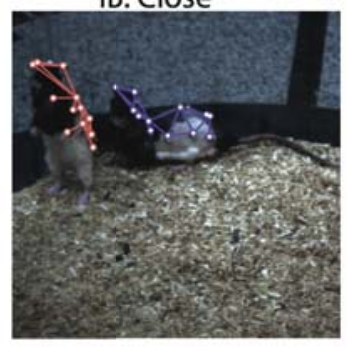

Mislabel

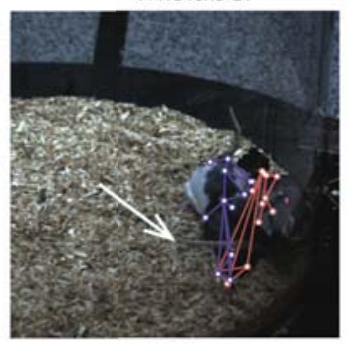

Figure 3: Example DANNCE predictions, reprojected onto a single camera view, for specific interaction behavioral categories. Example predictions are on validation recordings, with the validation animal (Subject 5) in red. Each frame is labeled with the interaction behavior category. The white arrow in the "Mislabel" panel points to an error in head identity prediction. Example Movies.

\begin{tabular}{llllllll}
\hline & MPJPE $_{H}$ & MPJPE $_{T}$ & MPJPE & PJPE $_{50}$ & PCK@ 0.5 & PCK@0.75 & mPCK \\
\hline DANNCE.L2* & 6.44 & 9.02 & 8.37 & 7.63 & 0.68 & 0.89 & 0.88 \\
DANNCE.L2 & 4.87 & 7.93 & 7.17 & 6.44 & 0.79 & 0.94 & 0.93 \\
DANNCE.L1* $^{*}$ & 4.28 & $\mathbf{7 . 1 3}$ & $\mathbf{6 . 4 1}$ & $\mathbf{5 . 7 7}$ & 0.83 & 0.96 & 0.95 \\
DANNCE.L1 & $\mathbf{4 . 2 2}$ & 7.35 & 6.57 & 5.87 & 0.83 & 0.96 & 0.95 \\
\hline
\end{tabular}

Table 2: DANNCE 3D multi-animal pose estimation benchmarks. In DANNCE. $X, X$ indicates the type of loss function used for training. $(*)$ was trained from a random initialization of weights, and the others from a network pre-trained on Rat 7M [18]. $\left(\mathrm{PJPE}_{50}\right)$ 50th percentile of the per joint prediction error (in $\mathrm{mm}$ ), i.e. the Euclidean distance between predicted and ground-truth markers. (MPJPE) mean PJPE, also broken down by head $\left(\mathrm{MPJPE}_{H}\right)$ and trunk $\left(\mathrm{MPJPE}_{T}\right)$. (PCK@0.5) percent correct keypoints using a distance threshold of $50 \%$ of the distance between two head markers. (PCK@0.75) PCK using a threshold of $75 \%$ of the distance. (mPCK) mean PCK over 11 equally spaced thresholds.

We trained DANNCE for 30 epochs, using 420k images (70k poses) per epoch, and varied the pretraining conditions and type of loss function to measure the influence of these parameters on performance. Our results on withheld validation subject 5 are presented in Table 2. When using DANNCE's previously published L2 loss function, DANNCE performance improved with pretraining on Rat 7M. However, training with an L1 loss, with or without pretraining, ultimately minimized the mean per joint prediction error (MPJPE) across all markers (additionally broken down by head, MPJPE $_{H}$, and trunk, MPJPE ${ }_{T}$ ) and maximized percent correct keypoints (PCK) at all distance thresholds (@ fractions of the distance between two head markers - $19.4 \mathrm{~mm}$ ). Across behaviors, DANNCE tracked Investigate with the smallest and CrouchExplore with the the largest error, respectively, although error was within 10\% across most behavioral categories (Appendix Table 3). DANNCE performed similarly well on all close social interaction behaviors (Appendix Table 4). Qualitatively, 
DANNCE generally made remarkably consistent landmark predictions even in periods of spatial overlap between animals, but it did sometimes briefly assign head landmarks to the wrong animal during specific close interaction poses (Fig. 3).

\section{Limitations}

Our dataset will already be a valuable resource for social behavioral tracking, but there are several present limitations that could be addressed in future work. First, due to frequent occlusions in the multi-animal settings, there are periods without accurate landmark tracking that we dropped from the dataset. Future datasets could incorporate a larger number of cameras to reduce the number of missing data periods. Second, the ground-truth motion capture data comes from a reduced 12-marker set that does not capture points on the distal limbs, and this could contribute to a loss of precision in behavioral identification. One potential solution for limb tracking is to train using a combination of the 20-marker Rat7M, which includes multiple limb markers, and PAIR-R24M datasets. Limb keypoints could also be added to the dataset using a combination of manual labeling, e.g. through crowdsourced annotation, and inference, similar to datasets like CMU Panoptic [25]. However, annotating keypoints in animals is generally more challenging for non-primate species, where identification of body parts requires more domain knowledge, making the use of crowd-sourced annotation platforms challenging.

\section{Discussion}

The PAIR-R24M dataset is the largest and most diverse benchmark dataset for the rapidly growing field of multi-animal behavioral measurement and analysis. We make the dataset available for researchers interested in training new multi-animal tracking and action recognition algorithms, and for researchers interested in mining the data for new quantitative insights on the nature of social behavior. Specifically, we expect that this dataset will help to address the problems of multi-animal $3 \mathrm{D}$ pose estimation and instance segmentation.

In our dataset we solve instance segmentation by identifying individuals using known differences in their respective marker sets. These ground-truth animal identities will assist in the development and evaluation of deep learning algorithms that identify individuals through either top-down inference, such as convolutional networks for identity detection or center-of-mass tracking (e.g. [20,59,60]), or bottom-up inference such as 3D extensions of part affinity fields [61].

The PAIR-R24M dataset should also help develop new approaches for multi-animal 3D pose estimation. Here, we performed pose estimation using a state-of-the-art volumetric animal pose tracking approach. While our approach was generally effective, it made mistakes on some types of close interaction, a relevant concern considering that most interesting social behaviors are characterized by profound animal-animal overlap and contorted poses. Our results may be improved by newer architectures that employ semi-supervised learning or temporal convolutions [56] in addition to previously discussed bottom-up methods. Additionally, while highly performant, the use of volumetric convolution is computationally expensive, limiting inference speeds. PAIR-R24M will aid the development and evaluation of new, fast and performant multi-view 3D pose estimation algorithms.

While the PAIR-R24M dataset is an important step in the collection and dissemination of benchmarks for animal pose estimation, it can be extended in many ways. While we used motion capture as a high-throughput means of collecting training data, labels for animal hands, feet, and other appendages will be necessary for training algorithms that predict more complete descriptions of animal movement. These labels could come from human annotators [18], and crowd-sourcing efforts have begun to assemble such detailed annotations for animals in 2D (e.g. [62]; although see Section 4). Datasets extending beyond keypoints to capture an animal's full 3D body surface, as is now possible in human subjects, will also be valuable. While 3D scans have been used to assemble parametric body models of animals in specific poses [63], the databases that are available are still small compared to those available in humans $[36,64]$ and do not contain data from freely moving subjects. While cross-domain adaptation approaches $[43,62,65]$ may facilitate some progress in 3D surface estimation, ground-truth databases are needed to appropriately benchmark and train these techniques. Finally, future datasets from other species, environments, and social contexts will help to build algorithms that are flexible across a rich array of tasks and contexts, with the ultimate goal of enabling methodologies for full reconstruction of animal kinematics in complex, occlusive environments, with as few as one camera. 


\section{Acknowledgements}

J.D.M. acknowledges support from the Helen Hay Whitney Foundation and NINDS (K99NS112597), D.E.A. from NSF (DGE1745303), B.P.Ö from SFARI (646706), NIH (R01GM136972), and the Starr Family Foundation., and T.W.D. from NIH (R01GM136972) and the McKnight Foundation Technological Innovations in Neuroscience Award.

\section{References}

[1] Edward O Wilson. Sociobiology: The new synthesis. Harvard University Press, 2000.

[2] Robin IM Dunbar and Susanne Shultz. Evolution in the social brain. Science, 317(5843):13441347, 2007.

[3] Stefano Porcelli, Nic Van Der Wee, Steven van der Werff, Moji Aghajani, Jeffrey C Glennon, Sabrina van Heukelum, Floriana Mogavero, Antonio Lobo, Francisco Javier Olivera, Elena Lobo, et al. Social brain, social dysfunction and social withdrawal. Neuroscience \& Biobehavioral Reviews, 97:10-33, 2019.

[4] Daniel P Kennedy and Ralph Adolphs. The social brain in psychiatric and neurological disorders. Trends in cognitive sciences, 16(11):559-572, 2012.

[5] Stacey J Sukoff Rizzo and Jacqueline N Crawley. Behavioral phenotyping assays for genetic mouse models of neurodevelopmental, neurodegenerative, and psychiatric disorders. Annиal Review of Animal Biosciences, 5:371-389, 2017.

[6] Jacqueline N Crawley. Mouse behavioral assays relevant to the symptoms of autism. Brain pathology, 17(4):448-459, 2007.

[7] Christian L Ebbesen and Robert C Froemke. Body language signals for rodent social communication. Current Opinion in Neurobiology, 68:91-106, 2021.

[8] Cristina Segalin, Jalani Williams, Tomomi Karigo, May Hui, Moriel Zelikowsky, Jennifer J. Sun, Pietro Perona, David J. Anderson, and Ann Kennedy. The mouse action recognition system (mars): a software pipeline for automated analysis of social behaviors in mice. bioRxiv, 2020.

[9] Weizhe Hong, Ann Kennedy, Xavier P Burgos-Artizzu, Moriel Zelikowsky, Santiago G Navonne, Pietro Perona, and David J Anderson. Automated measurement of mouse social behaviors using depth sensing, video tracking, and machine learning. Proceedings of the National Academy of Sciences, 112(38):E5351-E5360, 2015.

[10] Fabrice De Chaumont, Renata Dos-Santos Coura, Pierre Serreau, Arnaud Cressant, Jonathan Chabout, Sylvie Granon, and Jean-Christophe Olivo-Marin. Computerized video analysis of social interactions in mice. Nature Methods, 9(4):410-417, 2012.

[11] Kristin Branson, Alice A Robie, John Bender, Pietro Perona, and Michael H Dickinson. Highthroughput ethomics in large groups of drosophila. Nature Methods, 6(6):451-457, 2009.

[12] Ugne Klibaite and Joshua W Shaevitz. Paired fruit flies synchronize behavior: Uncovering social interactions in drosophila melanogaster. PLOS Computational Biology, 16(10):e1008230, 2020.

[13] Talmo D Pereira, Diego E Aldarondo, Lindsay Willmore, Mikhail Kislin, Samuel S-H Wang, Mala Murthy, and Joshua W Shaevitz. Fast animal pose estimation using deep neural networks. Nature Methods, 16(1):117, 2019.

[14] Jacob M Graving, Daniel Chae, Hemal Naik, Liang Li, Benjamin Koger, Blair R Costelloe, and Iain D Couzin. Deepposekit, a software toolkit for fast and robust animal pose estimation using deep learning. eLife, 8:e47994, 2019.

[15] Alexander Mathis, Pranav Mamidanna, Kevin M Cury, Taiga Abe, Venkatesh N Murthy, Mackenzie Weygandt Mathis, and Matthias Bethge. Deeplabcut: markerless pose estimation of user-defined body parts with deep learning. Nature Neuroscience, 21(9):1281-1289, 2018.

[16] Praneet C Bala, Benjamin R Eisenreich, Seng Bum Michael Yoo, Benjamin Y Hayden, Hyun Soo Park, and Jan Zimmermann. Automated markerless pose estimation in freely moving macaques with openmonkeystudio. Nature Communications, 11(1):1-12, 2020. 
[17] Christian Zimmermann, Artur Schneider, Mansour Alyahyay, Thomas S Brox, and Ilka Diester. Freipose: A deep learning framework for precise animal motion capture in $3 \mathrm{~d}$ spaces. bioRxiv, 2020.

[18] Timothy W Dunn, Jesse D Marshall, Kyle S Severson, Diego E Aldarondo, David GC Hildebrand, Selmaan N Chettih, William L Wang, Amanda J Gellis, David E Carlson, Dmitriy Aronov, et al. Geometric deep learning enables 3d kinematic profiling across species and environments. Nature Methods, pages 1-10, 2021.

[19] Zhe Cao, Tomas Simon, Shih-En Wei, and Yaser Sheikh. Realtime multi-person 2d pose estimation using part affinity fields. In Proceedings of the IEEE conference on computer vision and pattern recognition (CVPR), pages 7291-7299, 2017.

[20] Talmo D. Pereira, Nathaniel Tabris, Junyu Li, Shruthi Ravindranath, Eleni S. Papadoyannis, Z. Yan Wang, David M. Turner, Grace McKenzie-Smith, Sarah D. Kocher, Annegret L. Falkner, Joshua W. Shaevitz, and Mala Murthy. Sleap: Multi-animal pose tracking. bioRxiv, 2020.

[21] Jessy Lauer, Mu Zhou, Shaokai Ye, William Menegas, Tanmay Nath, Mohammed Mostafizur Rahman, Valentina Di Santo, Daniel Soberanes, Guoping Feng, Venkatesh N Murthy, et al. Multi-animal pose estimation and tracking with deeplabcut. bioRxiv, 2021.

[22] Daniel Joska, Liam Clark, Naoya Muramatsu, Ricardo Jericevich, Fred Nicolls, Alexander Mathis, Mackenzie W Mathis, and Amir Patel. Acinoset: A 3d pose estimation dataset and baseline models for cheetahs in the wild. arXiv preprint arXiv:2103.13282, 2021.

[23] Sinead Kearney, Wenbin Li, Martin Parsons, Kwang In Kim, and Darren Cosker. Rgbd-dog: Predicting canine pose from rgbd sensors. In Proceedings of the IEEE/CVF Conference on Computer Vision and Pattern Recognition (CVPR), pages 8336-8345, 2020.

[24] Jennifer J Sun, Tomomi Karigo, Dipam Chakraborty, Sharada P Mohanty, David J Anderson, Pietro Perona, Yisong Yue, and Ann Kennedy. The multi-agent behavior dataset: Mouse dyadic social interactions. arXiv preprint arXiv:2104.02710, 2021.

[25] Hanbyul Joo, Tomas Simon, Xulong Li, Hao Liu, Lei Tan, Lin Gui, Sean Banerjee, Timothy Godisart, Bart Nabbe, Iain Matthews, Takeo Kanade, Shohei Nobuhara, and Yaser Sheikh. Panoptic studio: A massively multiview system for social interaction capture. IEEE transactions on pattern analysis and machine intelligence, 41(1):190-204, 2016.

[26] Vasileios Belagiannis, Sikandar Amin, Mykhaylo Andriluka, Bernt Schiele, Nassir Navab, and Slobodan Ilic. 3D pictorial structures for multiple human pose estimation. In 2014 IEEE Conference on Computer Vision and Pattern Recognition, pages 1669-1676. IEEE, jun 2014.

[27] Dushyant Mehta, Oleksandr Sotnychenko, Franziska Mueller, Weipeng Xu, Srinath Sridhar, Gerard Pons-Moll, and Christian Theobalt. Single-shot multi-person 3d pose estimation from monocular rgb. In 3D Vision (3DV), 2018 Sixth International Conference on. IEEE, sep 2018.

[28] Timo von Marcard, Roberto Henschel, Michael Black, Bodo Rosenhahn, and Gerard Pons-Moll. Recovering accurate $3 \mathrm{~d}$ human pose in the wild using imus and a moving camera. In European Conference on Computer Vision (ECCV), sep 2018.

[29] Dushyant Mehta, Helge Rhodin, Dan Casas, Pascal Fua, Oleksandr Sotnychenko, Weipeng Xu, and Christian Theobalt. Monocular 3d human pose estimation in the wild using improved cnn supervision. In 3D Vision (3DV), 2017 Fifth International Conference on. IEEE, 2017.

[30] Long Chen, Haizhou Ai, Rui Chen, Zijie Zhuang, and Shuang Liu. Cross-view tracking for multi-human 3D pose estimation at over 100 FPS. In 2020 IEEE/CVF Conference on Computer Vision and Pattern Recognition (CVPR), pages 3276-3285. IEEE, jun 2020.

[31] Vinkle Srivastav, Thibaut Issenhuth, Abdolrahim Kadkhodamohammadi, Michel de Mathelin, Afshin Gangi, and Nicolas Padoy. Mvor: A multi-view rgb-d operating room dataset for $2 \mathrm{~d}$ and 3d human pose estimation. arXiv preprint arXiv:1808.08180, 2018.

[32] Gul Varol, Javier Romero, Xavier Martin, Naureen Mahmood, Michael J. Black, Ivan Laptev, and Cordelia Schmid. Learning from synthetic humans. In IEEE Conference on Computer Vision and Pattern Recognition (CVPR), pages 4627-4635. IEEE, jul 2017.

[33] Matthew Loper, Naureen Mahmood, Javier Romero, Gerard Pons-Moll, and Michael J. Black. Smpl. ACM transactions on graphics, 34(6):1-16, oct 2015. 
[34] Matteo Fabbri, Fabio Lanzi, Simone Calderara, Andrea Palazzi, Roberto Vezzani, and Rita Cucchiara. Learning to detect and track visible and occluded body joints in a virtual world. In European Conference on Computer Vision (ECCV), volume 11208, pages 450-466. Cham, 2018.

[35] Vasileios Choutas, Georgios Pavlakos, Timo Bolkart, Dimitrios Tzionas, and Michael J. Black. Monocular expressive body regression through body-driven attention. In European Conference on Computer Vision (ECCV), volume 12355, pages 20-40. 2020.

[36] Naureen Mahmood, Nima Ghorbani, Nikolaus F Troje, Gerard Pons-Moll, and Michael J Black. Amass: Archive of motion capture as surface shapes. In Proceedings of the IEEE/CVF International Conference on Computer Vision (ICCV), pages 5442-5451, 2019.

[37] Riza Alp Guler, Natalia Neverova, and Iasonas Kokkinos. Densepose: dense human pose estimation in the wild. In 2018 IEEE/CVF Conference on Computer Vision and Pattern Recognition (CVPR), pages 7297-7306. IEEE, jun 2018.

[38] Karim Iskakov, Egor Burkov, Victor Lempitsky, and Yury Malkov. Learnable triangulation of human pose. In 2019 IEEE/CVF International Conference on Computer Vision (ICCV), pages 7717-7726. IEEE, oct 2019.

[39] Tanmay Nath, Alexander Mathis, An Chi Chen, Amir Patel, Matthias Bethge, and Mackenzie Weygandt Mathis. Using DeepLabCut for 3D markerless pose estimation across species and behaviors. Nature Protocols, 14(7):2152-2176, jun 2019.

[40] Libby Zhang, Tim Dunn, Jesse Marshall, Bence Olveczky, and Scott Linderman. Animal pose estimation from video data with a hierarchical von mises-fisher-gaussian model. In Arindam Banerjee and Kenji Fukumizu, editors, Proceedings of The 24th International Conference on Artificial Intelligence and Statistics, volume 130 of Proceedings of Machine Learning Research, pages 2800-2808. PMLR, 13-15 Apr 2021.

[41] Pierre Karashchuk, Katie L Rupp, Evyn S Dickinson, Elischa Sanders, Eiman Azim, Bingni W Brunton, and John C Tuthill. Anipose: a toolkit for robust markerless 3d pose estimation. Cell Reports, 2021.

[42] Semih Günel, Helge Rhodin, Daniel Morales, João Campagnolo, Pavan Ramdya, and Pascal Fua. DeepFly3D, a deep learning-based approach for 3D limb and appendage tracking in tethered, adult drosophila. eLife, 8, oct 2019.

[43] Luis A Bolaños, Dongsheng Xiao, Nancy L Ford, Jeff M LeDue, Pankaj K Gupta, Carlos Doebeli, Hao Hu, Helge Rhodin, and Timothy H Murphy. A three-dimensional virtual mouse generates synthetic training data for behavioral analysis. Nature Methods, 18(4):378-381, apr 2021.

[44] Adam Gosztolai, Semih Günel, Victor Lobato Ríos, Marco Pietro Abrate, Daniel Morales, Helge Rhodin, Pascal Fua, and Pavan Ramdya. LiftPose3D, a deep learning-based approach for transforming two-dimensional to three-dimensional poses in laboratory animals. Nature Methods, aug 2021.

[45] Julieta Martinez, Rayat Hossain, Javier Romero, and James J. Little. A simple yet effective baseline for 3d human pose estimation. In 2017 IEEE International Conference on Computer Vision (ICCV), pages 2659-2668. IEEE, oct 2017.

[46] Markus Marks, Jin Qiuhan, Oliver Sturman, Lukas von Ziegler, Sepp Kollmorgen, Wolfger von der Behrens, Valerio Mante, Johannes Bohacek, and Mehmet Fatih Yanik. SIPEC: the deep-learning swiss knife for behavioral data analysis. BioRxiv, oct 2020.

[47] Simon RO Nilsson, Nastacia L. Goodwin, Jia Jie Choong, Sophia Hwang, Hayden R Wright, Zane C Norville, Xiaoyu Tong, Dayu Lin, Brandon S. Bentzley, Neir Eshel, Ryan J McLaughlin, and Sam A. Golden. Simple behavioral analysis (SimBA) - an open source toolkit for computer classification of complex social behaviors in experimental animals. BioRxiv, apr 2020.

[48] Fabrice de Chaumont, Elodie Ey, Nicolas Torquet, Thibault Lagache, Stéphane Dallongeville, Albane Imbert, Thierry Legou, Anne-Marie Le Sourd, Philippe Faure, Thomas Bourgeron, and Jean-Christophe Olivo-Marin. Real-time analysis of the behaviour of groups of mice via a depth-sensing camera and machine learning. Nature Biomedical Engineering, 3(11):930-942, may 2019. 
[49] Jumpei Matsumoto, Susumu Urakawa, Yusaku Takamura, Renato Malcher-Lopes, Etsuro Hori, Carlos Tomaz, Taketoshi Ono, and Hisao Nishijo. A 3D-video-based computerized analysis of social and sexual interactions in rats. Plos One, 8(10):e78460, oct 2013.

[50] Mayank Kabra, Alice A Robie, Marta Rivera-Alba, Steven Branson, and Kristin Branson. JAABA: interactive machine learning for automatic annotation of animal behavior. Nature Methods, 10(1):64-67, jan 2013.

[51] Ugne Klibaite, Gordon J Berman, Jessica Cande, David L Stern, and Joshua W Shaevitz. An unsupervised method for quantifying the behavior of paired animals. Physical Biology, 14(1):015006, 2017.

[52] Jesse D Marshall, Diego E Aldarondo, Timothy W Dunn, William L Wang, Gordon J Berman, and Bence P Ölveczky. Continuous whole-body $3 \mathrm{~d}$ kinematic recordings across the rodent behavioral repertoire. Neuron, 109(3):420-437, 2021.

[53] Gordon J Berman. Measuring behavior across scales. BMC Biology, 16(1):1-11, 2018.

[54] Xavier P Burgos-Artizzu, Piotr Dollár, Dayu Lin, David J Anderson, and Pietro Perona. Social behavior recognition in continuous video. In 2012 IEEE Conference on Computer Vision and Pattern Recognition (CVPR), pages 1322-1329. IEEE, 2012.

[55] Gordon J Berman, Daniel M Choi, William Bialek, and Joshua W Shaevitz. Mapping the stereotyped behaviour of freely moving fruit flies. Journal of The Royal Society Interface, 11(99):20140672, 2014.

[56] Dario Pavllo, Christoph Feichtenhofer, David Grangier, and Michael Auli. 3d human pose estimation in video with temporal convolutions and semi-supervised training. In Proceedings of the IEEE/CVF Conference on Computer Vision and Pattern Recognition (CVPR), June 2019.

[57] Eyrun Eyjolfsdottir, Kristin Branson, Yisong Yue, and Pietro Perona. Learning recurrent representations for hierarchical behavior modeling. arXiv preprint arXiv:1611.00094, 2016.

[58] Gordon J Berman, William Bialek, and Joshua W Shaevitz. Predictability and hierarchy in drosophila behavior. Proceedings of the National Academy of Sciences, 113(42):11943-11948, 2016.

[59] Tristan Walter and Iain D Couzin. Trex, a fast multi-animal tracking system with markerless identification, and 2d estimation of posture and visual fields. eLife, 10:e64000, 2021.

[60] Francisco Romero-Ferrero, Mattia G Bergomi, Robert C Hinz, Francisco JH Heras, and Gonzalo $\mathrm{G}$ de Polavieja. Idtracker. ai: tracking all individuals in small or large collectives of unmarked animals. Nature Methods, 16(2):179-182, 2019.

[61] Ding Liu, Zixu Zhao, Xinchao Wang, Yuxiao Hu, Lei Zhang, and Thomas Huang. Improving $3 \mathrm{~d}$ human pose estimation via 3d part affinity fields. In 2019 IEEE Winter Conference on Applications of Computer Vision (WACV), pages 1004-1013. IEEE, 2019.

[62] Jinkun Cao, Hongyang Tang, Hao-Shu Fang, Xiaoyong Shen, Cewu Lu, and Yu-Wing Tai. Cross-domain adaptation for animal pose estimation. In The IEEE International Conference on Computer Vision (ICCV), October 2019.

[63] Silvia Zuffi, Angjoo Kanazawa, David Jacobs, and Michael J. Black. 3D menagerie: Modeling the 3D shape and pose of animals. In Proceedings IEEE Conference on Computer Vision and Pattern Recognition (CVPR) 2017, pages 5524-5532. IEEE, July 2017.

[64] Kathleen M Robinette, Hans Daanen, and Eric Paquet. The caesar project: a 3-d surface anthropometry survey. In Second International Conference on 3-D Digital Imaging and Modeling (Cat. No. PR00062), pages 380-386. IEEE, 1999.

[65] Artsiom Sanakoyeu, Vasil Khalidov, Maureen S McCarthy, Andrea Vedaldi, and Natalia Neverova. Transferring dense pose to proximal animal classes. In Proceedings of the IEEE/CVF Conference on Computer Vision and Pattern Recognition, pages 5233-5242, 2020.

[66] Jack Bandy and Nicholas Vincent. Addressing" documentation debt" in machine learning research: A retrospective datasheet for bookcorpus. arXiv preprint arXiv:2105.05241, 2021. 


\section{Checklist}

1. For all authors...

(a) Do the main claims made in the abstract and introduction accurately reflect the paper's contributions and scope? [Yes]

(b) Did you describe the limitations of your work? [Yes] See Section 4

(c) Did you discuss any potential negative societal impacts of your work? [Yes] Please see the supplemental impact statement (Appendix 2).

(d) Have you read the ethics review guidelines and ensured that your paper conforms to them? [Yes]

2. If you are including theoretical results...

(a) Did you state the full set of assumptions of all theoretical results? [N/A]

(b) Did you include complete proofs of all theoretical results? [N/A]

3. If you ran experiments (e.g. for benchmarks)...

(a) Did you include the code, data, and instructions needed to reproduce the main experimental results (either in the supplemental material or as a URL)? [Yes] Please see Appendix 5.

(b) Did you specify all the training details (e.g., data splits, hyperparameters, how they were chosen)? [Yes] Yes, this information is included in the Results and in Appendix 5.

(c) Did you report error bars (e.g., with respect to the random seed after running experiments multiple times)? [Yes] We did not run experiments multiple times, but we reported multiple measures of the statistical distributions of error metrics in Table 2.

(d) Did you include the total amount of compute and the type of resources used (e.g., type of GPUs, internal cluster, or cloud provider)? [Yes] This information is reported in Appendix 5.

4. If you are using existing assets (e.g., code, data, models) or curating/releasing new assets...

(a) If your work uses existing assets, did you cite the creators? [N/A]

(b) Did you mention the license of the assets? [N/A] Yes, the CC BY 4.0 license is included in the datasheet (Appendix 1) and in our repositories.

(c) Did you include any new assets either in the supplemental material or as a URL? [Yes] Yes, the dataset is available at figshare as detailed in the Datasheet (Appendix 1).

(d) Did you discuss whether and how consent was obtained from people whose data you're using/curating? [N/A] We collected the data ourselves from animals, following protocols for animal care approved by the Harvard University IACUC (Appendix 2).

(e) Did you discuss whether the data you are using/curating contains personally identifiable information or offensive content? [N/A] The data come from animals and thus have no personally identifiable information of offensive content.

5. If you used crowdsourcing or conducted research with human subjects...

(a) Did you include the full text of instructions given to participants and screenshots, if applicable? [N/A]

(b) Did you describe any potential participant risks, with links to Institutional Review Board (IRB) approvals, if applicable? [N/A]

(c) Did you include the estimated hourly wage paid to participants and the total amount spent on participant compensation? [N/A] 


\section{Appendix 1 I Dataset Nutrition Label}

\begin{tabular}{|c|c|}
\hline \multicolumn{2}{|c|}{$\begin{array}{l}\text { Dataset PAIR-R24M } \\
\text { Number of Frames } 24 \text { Million } \\
\text { Number of Annotated Animals } 7 \\
\text { Number of Unannotated Animals } 2\end{array}$} \\
\hline \multicolumn{2}{|l|}{ Metadata } \\
\hline $\begin{array}{l}\text { Filename } \\
\text { Format } \\
\text { URL https://figsha } \\
\text { DOI } \\
\text { Keywords } \\
\text { Rows } \\
\text { Columns } \\
\text { Missing Data } \\
\text { License } \\
\text { First Released }\end{array}$ & $\begin{array}{r}\text { README.txt } \\
\text {.mp4, .csv, .json } \\
\text { om/articles/dataset/PAIRS_dataset/14754374 } \\
\text { s://doi.org/10.6084/m9.figshare.14754374.v2 } \\
\text { al Behavior, Pose Estimation, Social Behavior } \\
\text { Timepoints } \\
\text { 3D keypoint positions, behavior labels } \\
\text { Stored as NaN } \\
\text { CC BY } 4.0 \\
\text { June } 72021\end{array}$ \\
\hline \multicolumn{2}{|l|}{ Variables | markerDataset } \\
\hline $\begin{array}{l}\text { center_of_mass } \\
\text { aligned_position } \\
\text { absolute_position } \\
\text { goodFrame } \\
\text { behavior } \\
\text { interactionCategory }\end{array}$ & $\begin{array}{r}\text { Center of mass of the animal } \\
\text { Marker positions aligned to center of mass } \\
\text { Marker positions in global arena coordinates } \\
\text { Frames without missing markers } \\
\text { Behavior of the animal } \\
\text { Interaction category of the animal pair }\end{array}$ \\
\hline \multicolumn{2}{|l|}{ Variables | Calibration } \\
\hline $\begin{array}{l}\text { rotationMatrix } \\
\text { translationMatrix } \\
\text { intrinsicMatric } \\
\text { radialDistortion } \\
\text { tangentialDistortion }\end{array}$ & $\begin{array}{r}\text { Rotation Matrix of Camera } \\
\text { Translation Matrix of Camera } \\
\text { Intrinsic Matrix of Camera } \\
\text { Rotational Distortion Coefficient } \\
\text { Translational Distortion Coefficient }\end{array}$ \\
\hline
\end{tabular}

Figure 4: PAIR-R24M nutrition label, constructed using the template from Bandy et al. [66]. Note that the exact DOI may change as the dataset is updated. 


\section{Appendix 2 I Impact and Animal Care Statement}

Preclinical screening of animal models is a crucial step in the drug discovery pipeline, and developing improved social assays thus represents an important step to alleviating human disease burden. Careful measurements and associated analysis frameworks to understand the natural behavior of animals in 3D should facilitate new approaches for animal phenotyping and contribute to the development of new theraputics, especially in the cases of neuropsychiatric diseases that affect social behaviors, such as Autism Spectrum Disorders, Williams syndrome, and schizophrenia. All experiments were performed at Harvard's AAALAC-accredited animal facility. The care and experimental manipulation of all animals were reviewed and approved by the Harvard University Faculty of Arts and Sciences Institutional Animal Care and Use Committee. All surgical procedures were designed to limit pain and discomfort. More details on the experimental procedures are given in [52]. 


\section{Appendix 3 I Discrepancies in Motion Capture and Video Tracking}
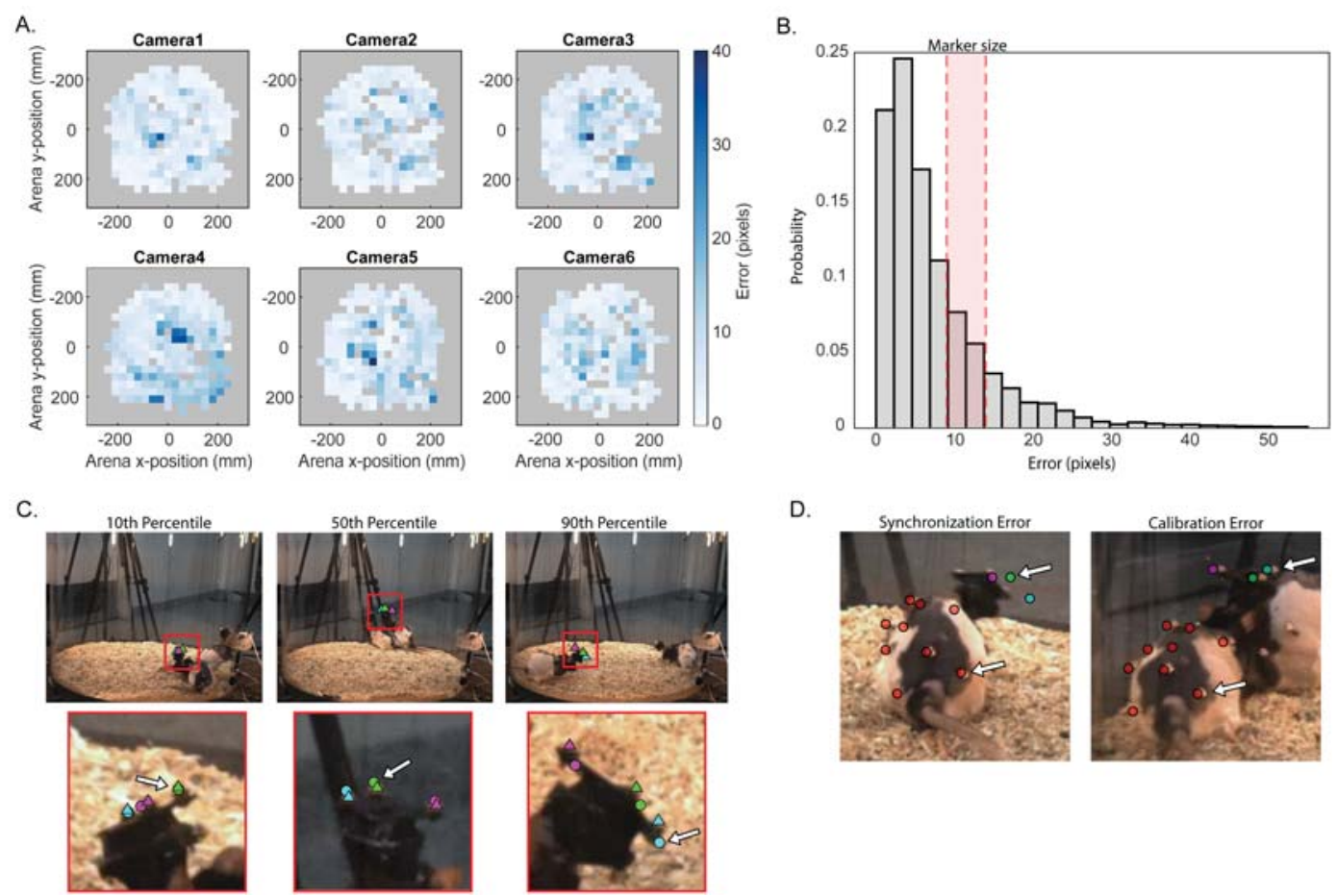

D.

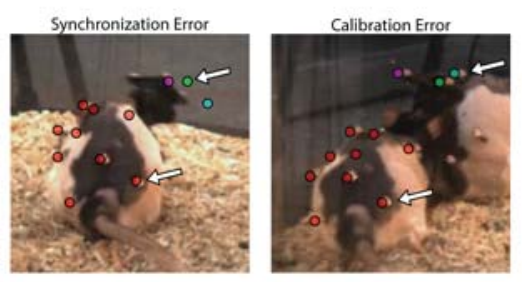

Figure 5: (A) 2D histograms of the discrepancy in the position of the three markers on an animal's head between hand labels and motion capture. Heatmaps depict the differences in pixels between the projections of the motion capture data into 2D and the human hand-labeled points as a function of the $\mathrm{X}$ - and $\mathrm{y}$ - position of the headcap markers in the arena, for a single camera view. (B) Histogram of the discrepancy in pixels across all cameras in all views. Errors range from 0.04 to 50.1 pixels (px), with a mean error of $7.1 \mathrm{px}$ (or around $2.7 \mathrm{~mm}$ ). The exact pixel size of the retroreflective marker ( $5 \mathrm{~mm}$ in diameter) depends on the camera view and is indicated by the shaded red bar. (C) Three example frames (top) showing the 10th percentile (left; error $=1.3 \mathrm{px}$ ), 50th percentile (center; error $=4.8 \mathrm{px}$ ), and 90th percentile (right; error $=15.7 \mathrm{px}$ ) discrepancy from camera 5 in $(\mathrm{A})$. The white arrows in the zoomed in images (bottom) highlight the marker representing the respective percentile. (D) An example of synchronization error (left) and calibration error (right) with arrows pointing to a head marker and a body marker for comparison. In the frame with synchronization error, the head markers show larger error than the body markers, likely due to the animal moving its head quickly and the RGB video lagging behind. In the frame with calibration error, the head and body marker errors are more uniform, making an issue with calibration parameters more likely.

In a subset of video frames and camera views, we observed a discrepancy between the marker positions, as tracked using motion capture, and the apparent marker positions in the video frames. Such a discrepancy could be caused by either noise camera calibration, or temporally localized variability in RGB video camera synchronization with motion capture. To quantify the magnitude and extent of these discrepancies, we hand labeled the position of the markers on the head in 2078 video frames and compared them with the projections of points tracked using motion capture. Differences varied across cameras and positions of the animal in the arena (Fig. 5A). On average, differences (7 px mean, 5 px median) were well below both the marker size (9-14 px) and measured precision of hand-labelers (12 px [52]; Fig. 5B-C). Nevertheless, on $10 \%$ of frames these differences were greater than the marker diameter, although they rarely exceeded two marker diameters $(\sim 1 \%$ of frames $)$. Motion capture discrepancies appeared notably smaller for markers on the body, which are less sensitive to slight variability in synchronization (Fig. 5D). Discrepancies are nearly unavoidable in large datasets [38], and can in principle add robustness to 3D markerless pose detection models [18]. Nevertheless, these deviations may present a noise ceiling for 3D pose tracking, and could be removed, if desired, when running benchmarks [38]. 


\section{Appendix 4 I Constant Head Segment Lengths Suggest Accurate Animal Identity Tracking}



Figure 6: Normalized histograms of head segment lengths for Subjects 1-5, measured from all recorded motion capture data and broken down by recording type: paired recordings in which only one subject had markers (red lines) and paired recordings in which both subjects had markers (blue lines). For each subject, histograms for each of the three head segments are plotted together on one graph.

Motion capture measurements are so precise that they enable fingerprinting of each subject via quantification of small subject-specific differences in head segment lengths; these differences arise from variability in marker placement during headcap construction. We established reference head segment lengths for each subject by examining their distributions in marker + markerless recordings, where identity swapping is impossible. In marker + marker recordings, swaps in animal identity should manifest as frames with head segment lengths deviating from each animal's reference. We see little support for such swaps in the data. 


\section{Appendix 5 I DANNCE Training and Evaluation}

\begin{tabular}{llllllllllll}
\hline & B1 & B2 & B3 & B4 & B5 & B6 & B7 & B8 & B9 & B10 & B11 \\
\hline DANNCE.L2* & 7.82 & 8.24 & 8.10 & 8.08 & 8.84 & 8.24 & 8.25 & 8.23 & 10.16 & 8.12 & 8.21 \\
DANNCE.L2 & 7.08 & 6.53 & 6.96 & 7.21 & 7.31 & 6.67 & 7.64 & 7.45 & 9.82 & 7.27 & 7.08 \\
DANNCE.L1* & $\mathbf{6 . 2 8}$ & $\mathbf{6 . 0 3}$ & $\mathbf{6 . 2 1}$ & $\mathbf{6 . 4 0}$ & $\mathbf{6 . 5 8}$ & $\mathbf{5 . 8 7}$ & $\mathbf{6 . 7 8}$ & $\mathbf{6 . 4 9}$ & 9.78 & 6.38 & 6.34 \\
DANNCE.L1 & 6.46 & 6.19 & 6.26 & 6.56 & 6.77 & 6.12 & 6.97 & 6.74 & $\mathbf{9 . 3 9}$ & $\mathbf{6 . 3 2}$ & 6.34 \\
\hline
\end{tabular}

Table 3: MPJPE in validation subject 5, broken down by individual behavioral category.

\begin{tabular}{llll}
\hline & IB1 & IB2 & IB3 \\
\hline DANNCE.L2* & 8.68 & 9.30 & 8.57 \\
DANNCE.L2 & 7.44 & 8.24 & 7.26 \\
DANNCE.L1* & $\mathbf{6 . 6 1}$ & $\mathbf{7 . 2 2}$ & 6.56 \\
DANNCE.L1 & 6.74 & 7.58 & $\mathbf{6 . 4 7}$ \\
\hline
\end{tabular}

Table 4: MPJPE in validation subject 5, broken down by interaction behavioral category.

Multi-animal DANNCE (https://github.com/spoonsso/dannce/) training and evaluation was performed in Python 3.7 using tensorflow (for the network) and pytorch (for parallel 3D volume generation). For efficiency, we trained multi-animal DANNCE using 4 NVIDIA V100 16 GB GPUs on the Harvard Odyssey compute cluster. We used training frames and ground-truth poses from 4 unique animal pairs, distributed over 7 1-hour recordings at $30 \mathrm{~Hz}$. To form the training set, 10,000 time points $(60,000$ frames) were sampled randomly without replacement from the time points in each recording having a complete motion capture marker set without imputation, resulting in 70,000 training samples total. We chose at the outset to train each DANNCE network for 30 epochs using a batch size of 4, and at the end of training we evaluated the performance of each network on the full validation dataset just once (results in Table 2, 3, 4). For the benchmarks presented here, we used all samples from a 1-hour recording of subject 3 and 5, evaluated over withheld validation subject 5 only, that had a complete motion capture marker set without imputation (43,285 samples; 259,710 frames). For each animal, we anchored its image volume to the 3D position of its "SpineM" marker in each frame.

For the benchmarks, we varied the loss function used for DANNCE training, using either mean squared error (L2) or mean absolute error (L1). We also tested training DANNCE from a random weight initialization, or from previously published weights found by training over images of single animals behaving in the Rat 7M dataset (https://github.com/spoonsso/dannce/) [18]. In all cases, we used DANNCE in the "AVG" architecture configuration (a 3D U-Net with a soft-argmax output layer) and trained using the Adam optimizer with $1 r=0.001$ and default parameters. We list the full set of DANNCE training parameters used in Table 5. Full architecture details and parameter definitions can be found on the dannce github.

To quantify DANNCE performance, we calculated standard 3D pose estimation error metrics, using a Procruste's alignment to ground-truth before calculations (translation and rotation only; no scaling). MPJPE was calculated as the mean Euclidean error across all markers after alignment. PJPE $_{50}$ is the median error across all markers. PCK metrics reflect accuracy over all markers after binarizing all predictions using the indicated threshold distances, expressed as fractions of the distance between two Head markers $(19.4 \mathrm{~mm})$. For the mPCK metric, we calculated PCK for each threshold in [0.5, $0.55,0.6,0.65,0.7,0.75,0.8,0.85,0.9,0.95,1]$ and took the mean. 


\begin{tabular}{ll}
\hline Parameter & Value \\
\hline nvox & 64 \\
n_channels_in & 3 \\
n_views & 6 \\
n_channels_out & 20 \\
new_last_kernel_size & {$[333]$} \\
batch_size & 4 \\
epochs & 30 \\
loss & 'mask_nan_keep_loss', 'mask_nan_11_loss' \\
lr & '1e-3' \\
net & 'unet3d_big_expectedvalue' \\
n_layers_locked & 0 \\
num_train_per_exp & 10000 \\
vmin & -120 \\
vmax & 120 \\
interp & 'nearest' \\
rotate & 1 \\
expval & 1 \\
channel_combo & 'None' \\
n_rand_views & 6 \\
predict_mode & 'torch' \\
data_split_seed & 11516 \\
depth & 0 \\
augment_continuous_rotation & 0 \\
mono & 0 \\
augment_hue & 0 \\
drop_landmark & 'None' \\
raw_im_h & 1048 \\
raw_im_w & 1328 \\
mirror & 0 \\
n_instances & 1 \\
write_npy & 'None' \\
\hline
\end{tabular}

Table 5: Values of DANNCE training parameters. 